\title{
Cornelia Raub-Kübne
}

\section{Nationalsozialismus und Kommunismus auf dem katholischen Land}

„Je ländlicher, je geschlossener protestantisch der Bezirk, je kleiner das Dorf: um so größer der Gewinn der Nationalsozialisten", behauptete 1931 das katholische Sonntagsblatt für die Diözese Speyer ${ }^{1}$ und skizzierte mit dieser auf die Nordpfalz und das angrenzende Hessen gemünzten Beschreibung durchaus typische Verhältnisse im damaligen Reich. Der „idealtypische Wähler der nationalsozialistischen Partei“, so fand auch die Forschung heraus, war „im Jahre 1932 [...] ein selbständiger protestantischer Angehöriger der Mittelklasse, der entweder auf einem Hof oder in einer kleinen Ortschaft lebte".2 Soziologische Klassiker ebenso wie neuere wahlhistorische Untersuchungen und alle ernst zu nehmenden Regionalstudien zum Katholizismus in der Weimarer Republik bestätigen, „daß kein anderes Sozialmerkmal die nationalsozialistischen Wahlerfolge so stark beeinflußt hat wie die Konfession“ und daß das bis zur nationalsozialistischen „Machtübernahme für das Land ebenso galt wie für die Städte". ${ }^{3}$ In einer Untersuchung der ländlichen Gemeinden in Baden und im Elsaß gelangte der französische Historiker Alfred Wahl zu dem Ergebnis: „Le nazisme était bien un phénomène des agriculteurs et des couches moyennes protestantes, du moins sur le plan électoral“"

Was dagegen das Verhältnis der bäuerlichen katholischen Bevölkerung zum Kommunismus anbelangt, so ist das jüngst von Klaus Michael Mallmann gezogene Fazit in seinem Buch über „Kommunisten in der Weimarer Republik“ aufschluß-

1 Der Christliche Pilger. Sonntagsblatt für das Bistum Speyer, Nr. 5, 1. Februar 1931, zitiert nach Thomas Fandel, Konfession und Nationalsozialismus. Evangelische und katholische Pfarrer in der Pfalz 1930-1939, Paderborn u.a. 1997, S. 24, Anm. 11.

2 Seymor M. Lipset, Der „Faschismus“, die Linke, die Rechte und die Mitte, in: Ernst Nolte (Hrsg.), Theorien über den Faschismus, Köln 1967, S. 449-491, hier S. 463.

3 Jürgen Falter, Hitlers Wähler, München 1991, S. 177, zum konfessionsabhängig unterschiedlichen Erfolg der NSDAP in ländlichen Gegenden, S. 181. Mit dem Anspruch, „zu einer Revision der Forschungsmethode und des Problemansatzes" beizutragen und "die ,orthodoxe" historiographische These" zu hinterfragen, nach der angeblich "die katholische Bevölkerung in der Weimarer Republik dem Nationalsozialismus gegenüber ,immun" war", tritt Oded Heilbronner an. Die zentralen Thesen seiner 1991 an der Hebrew University Jerusalem eingereichten Dissertation wurden durch mehrere renommierte Fachzeitschriften (Geschichte und Gesellschaft, Journal of Contemporary History, Social History) publik gemacht und von der Forschung unkritisch rezipiert. Wie jedoch anhand der neuerdings verfügbaren deutschen Fassung der Gesamtstudie festzustellen ist, verdienen Heilbronners widersprüchliche Behauptungen (vgl. z. B. S. 14, S. 73 f. u. S. 75 zum Wahlverhalten der deutschen Katholiken) und die schwach belegte These des angeblichen Niedergangs des bürgerlichen Vereinswesens als entscheidender Auslöser der Radikalisierung große Skepsis. Am meisten muß erstaunen, daß man in den wiedergegebenen Wahlergebnissen des Mikrobereichs Angaben zur konfessionellen Zusammensetzung der untersuchten Einheiten durchweg vermißt. Ders, Die Achillesferse des deutschen Katholizismus. Aus dem Hebräischen v. Matthias Schmidt, Gerlingen 1998.

4 Alfred Wahl, Confession et Comportement dans les Campagnes d'Alsace et de Bade 1871-1939. Catholiques, Protestants et Juifs. Démographie, Dynamisme Economique et Social, Relation et Attitude Politique. Thèse pour le Doctorat d'État (sous la direction de Raymond Poidevin), Metz 1980, S. 1231. 
reich: „Prima facie [...] waren rein protestantisches Terrain, hoher Industrialisierungs- und Urbanisierungsgrad sowie verwurzelte sozialdemokratische Traditionen [...] der ideale Boden für den deutschen Kommunismus" 5 . Auch dieser Befund wird durch kleinräumig konzipierte Studien erhärtet. Siegfried Weichlein, der das kommunistische Milieu im hessischen Landkreis Hanau untersucht hat, gelangt zu dem Ergebnis, „die explosive Mischung von Arbeitslosigkeit und katholischer Sozialisation förderte das Wachstum der KPD unter katholischen Arbeiterwählern. So blieb die KPD in erster Linie eine Partei der Industriegemeinden, ihre Bauernagitation in den agrarischen Landgemeinden stieß nur auf geringe Resonanz"6.

Indirekt läßt sich aus all diesen Feststellungen entnehmen: das katholische Land gilt der Forschung als Hort politischer Stabilität und Ort sozialer Beharrung, wo auch in den Krisenjahren der ersten deutschen Demokratie weder die NSDAP noch die radikale Linke große Erfolge feiern konnten. Offenbar bieten katholisch-ländliche Regionen einigermaßen schwierige Bedingungen, um Ursachen, Verlauf und Auswirkung der politischen Radikalisierung der deutschen Gesellschaft in der Weimarer Republik zu untersuchen. Daß es sich dennoch lohnen könnte, diese katholischen Agrargebiete einmal näher zu erforschen, um den vielschichtigen Ursachen der Krise des Weimarer Staates zu Beginn der 1930er Jahre auf den Grund gehen zu können, hoffe ich im folgenden deutlich machen zu können. Ich gebe zunächst einen kurzen Überblick zur Forschungslage zum katholischen Milieu. In einem zweiten Schritt stelle ich inhaltliche Ergebnisse der Forschung vor, die über kulturelle, soziale und politische Faktoren des Erfolgs oder Mißerfolgs der radikalen Weimarer Parteien auf dem katholischen Lande orientieren sollen. Dabei stütze ich mich vor allem auf Ergebnisse zum Land Baden, das nicht zufällig besser erforscht ist als andere katholische Regionen. Denn in Baden, dessen Industrialisierungsgrad in etwa dem des Reiches entsprach, wohnte noch etwa ein Drittel der Bevölkerung auf dem Lande meist in konfessionell weitgehend homogenen, sehr kleinen Orten mit weniger als 1000 Einwohnern. Als Folge der historischen Zersplitterung der Herrschaftsrechte war es landesweit zu einer auffälligen konfessionellen Durchmischung gekommen. Gemeinden mit gleichen sozioökonomischen Rahmenbedingungen, doch von verschiedener konfessioneller Zusammensetzung, grenzten häufig aneinander. Der Katholikenanteil lag in Baden 1925 bei 58 Prozent. Damit war der Freistaat deutlich „katholischer" als das Reich (31 Prozent). Trotzdem setzte die Radikalisierung der Wählerschaft hier früher ein. Diese Beobachtungen bieten der historischen Forschung günstige Ansatzpunkte, zumal auch die wahlstatistische Überlieferung für Baden besser ist als für viele anderen Regionen7. Ich will jedoch versuchen, Probleme anzusprechen,

5 Klaus-Michael Mallmann, Kommunisten in der Weimarer Republik. Sozialgeschichte einer revolutionären Bewegung, Darmstadt 1996.

6 Siegfried Weichlein, Sozialmilieus und politische Kultur in der Weimarer Republik. Lebenswelt, Vereinskultur, Politik in Hessen, Göttingen 1996, S. 292.

7 Vgl. Ellsworth Faris, Takeoff Point for the National Socialist Party. The Landtag Election in Baden 1929, in: CEH 8 (1975), S. 140-171, hier S. 142; Jürgen Falter/Hartmut Bömermann, Die unterschiedlichen Wahlerfolge der NSDAP in Baden und Württemberg: Ergebnis differenzierter Sozialstruktur oder regionalspezifischer Faktoren? in: Dieter Oberndörfer/Karl Schmitt (Hrsg.), Parteien und regionale politische Traditionen in der Bundesrepublik Deutschland, Berlin 1991, S. 283298. Da Falter/Bömermann den Anteil der Landbevölkerung in Gemeinden unter 5000 Einwoh- 
die nicht nur regional von Bedeutung sind, sondern allgemein interessierende $\mathrm{Zu}$ sammenhänge betreffen. Neben offenen Fragen werden auch methodische Überlegungen angestellt, die ein Leitfaden für künftige Forschungen sein könnten.

Unter „katholischem Land" werden im folgenden Gemeinden verstanden, die bis zu 2000 Einwohner zählten ${ }^{8}$ und nach ihrer konfessionellen Zusammensetzung zu mindestens 70 Prozent katholisch waren9. Arbeiterlandgemeinden, in denen die Erwerbsstruktur von benachbarten Industriestädten geprägt war, fallen unter diese Definition ebenso wie Dörfer mit vorherrschend agrarischer Lebensweise. Doch soll es hier nicht um Bergarbeitergemeinden in Industrieregionen wie dem Ruhrgebiet oder dem Saargebiet gehen, in denen eine spezifische Arbeiterkultur herrschte und die relativ gut erforscht sind.10 Statt dessen beziehen sich meine Ausführungen auf die katholischen Agrargebiete im Westen und Südwesten des Deutschen Reiches ${ }^{11}$.

Den eingangs zitierten Beobachtungen entsprechend, hat die Forschung zur Weimarer Republik den katholisch ländlichen Gebieten bisher nur geringe Aufmerksamkeit geschenkt. Zwar fand der lange vernachlässigte „,Mikro'bereich des Weimarer politisch-sozialen Systems" ${ }^{12}$ seit Beginn der 1990er Jahre in der historischen Forschung zunehmend Beachtung. Es entstanden auch zu katholischen Regionen eine Reihe von Milieustudien, die häufig längere Zeiträume umschlossen und die politischen Zäsuren von 1918, 1933 oder 1945 überspannten, um längerfristigen Trends wirtschaftlichen, sozialen, politischen oder kulturellen Wandels nachgehen zu können. Dabei überwog eindeutig das Interesse an solchen Regionen, in denen schneller sozialer Wandel und vorindustrielle politisch-soziale Leitbilder aufeinander trafen und sich die Frage aufdrängte, wie dies bewältigt

nern vergleichen, gelangen sie zu einer gegenüber dem Reich deutlich ländlicher geprägten Sozialstruktur in Baden.

8 In Gemeinden dieser Größenklasse lebte 1933 ein Drittel der Reichsbevölkerung. Der Anteil unter den deutschen Katholiken dürfte noch höher gewesen sein. Dietmar Petzina/Werner Abelshauser/ Anselm Faust, Sozialgeschichtliches Arbeitsbuch, Bd. 3. Materialien zur Statistik des Deutschen Reiches 1914-1945, München 1978, S. 36f.

9 Vgl. die analoge Definition des protestantischen Landes bei: Wolfram Pyta, Dorfgemeinschaft und Parteipolitik 1918-1933. Die Verschränkung von Milieu und Parteien in den protestantischen Landgebieten Deutschlands in der Weimarer Republik, Düsseldorf 1996, S. $14 \mathrm{f}$.

10 Klaus-Michael Mallmann/Horst Steffens, Lohn der Mühen, Geschichte der Bergarbeiter an der Saar, München 1989; Gerhard Paul, Die NSDAP des Saargebietes 1920-1935. Der verspätete Aufstieg der NSDAP in der katholisch-proletarischen Provinz, Saarbrücken 1987; ders./Klaus-Michael Mallmann, Milieus und Widerstand. Eine Verhaltensgeschichte der Gesellschaft im Nationalsozialismus, Bonn 1995; Klaus Tenfelde, Bergarbeiterkultur in Deutschland. Ein Überblick, in: Geschichte und Gesellschaft 5 (1979), S. 12-53; ders., Proletarische Provinz, Radikalisierung und Widerstand in Penzberg/Oberbayern 1900-1945, in: Martin Broszat/Elke Fröhlich/Anton Grossmann (Hrsg.), Bayern in der NS-Zeit, Bd.4, München 1981, S. 1-382; Michael Zimmermann, Schachtanlage und Zechenkolonie. Leben, Arbeit und Politik in einer Arbeitersiedlung 1880-1930, Essen 1987; Instruktiv, jedoch auf Bergarbeitergemeinden über 10000 Einwohner beschränkt: Wolfgang Jäger, Bergarbeitermilieus und Parteien im Ruhrgebiet. Zum Wahlverhalten des katholischen Bergarbeitermilieus bis 1933, München 1996.

11 Zu den sechs wichtigsten Agrarregionen des Deutschen Reichs: William Brustein, Logic of Evil. The Social Origins of the Nazi Party 1925-1933, New Haven u.a. 1996, S. $64 \mathrm{f}$. Ausgedehnte katholische Gebiete wiesen darunter nur zwei Regionen auf: das rheinisch-westfälische Agrargebiet und Süddeutschland mit Teilen Hessens, Badens, Bayerns und Württembergs.

12 Klaus Megerle/Peter Steinbach, Politische Kultur in der Krise. Neuere Beiträge zur Bestimmung der politischen Kultur in der Weimarer Republik, Teil 1, in: PVS-Literatur 22 (1981), S. 123-157, hier S. 137. 
wurde. Vielfach bediente man sich dabei des von dem Soziologen M. Rainer Lepsius für die Geschichtswissenschaft fruchtbar gemachten Milieukonzepts ${ }^{13}$. Lepsius konstatierte eine aus der historischen Entwicklung des 19. Jahrhunderts herrührende Spaltung der deutschen Gesellschaft in vier sozial-moralische Milieus. Darunter verstand er lebensweltliche Gesinnungsgemeinschaften unterschiedlicher sozialer Zusammensetzung, regionaler Tradition und parteipolitischer Präferenz. Diesem Konzept zufolge waren die politischen Parteien ihrem Selbstverständnis nach gewissermaßen die Aktionsausschüsse ihres jeweiligen Milieus. Bereitschaft oder Unvermögen der Parteien, Koalitionen zu bilden und Kompromisse einzugehen, stellten sich in dieser Perspektive als Reflex auf lebensweltlich ausgetragene Spannungen oder gar Konflikte zwischen den Milieus dar.

Über die Herausbildung und den Ausbau des katholischen Milieus im Kontext urbaner Lebensverhältnisse sind wir mittlerweile durch eine ganze Reihe von methodisch komplex angelegten Studien zur politischen Sozialgeschichte gut informiert. An neueren Arbeiten zu nennen sind insbesondere die milieuvergleichenden Studien von Siegfried Weichlein, der das katholische Milieu am Beispiel Fuldas analysiert und Antonius Liedhegeners vergleichende Studie zu Bochum und Münster, die weit ins 19. Jahrhundert zurückreicht und die Konstituierung und Verfestigung katholischer Milieus im Urbanisierungsprozeß konfessionell inhomogener großer Städte untersucht. Auch die von mir verfaßte Lokalstudie zur konfessionell gemischten, industriell geprägten badischen Kleinstadt Ettlingen ist hier zu erwähnen, in der Entstehung und Interaktion von drei sozialmoralischen Milieus vom 19. Jahrhundert bis zum Beginn des Zweiten Weltkriegs verfolgt werden ${ }^{14}$. So wissen wir manches darüber, wie städtisch-katholische Milieus entstanden und funktionierten, sich im Modernisierungsprozeß veränderten und (unter anderem während der Weimarer Republik) das politisch-soziale Verhalten der Milieuangehörigen beeinflußten.

$\mathrm{Daß}$ demgegenüber die ländliche Gesellschaft mit vorherrschend agrarischer Lebensweise, in der "das Politische“, mit einem Wort Martin Broszats gesprochen, seit je her "als Nebensache“ galt, seitens der Weimar-Forschung nur geringe Aufmerksamkeit erlangt hat, ist vermutlich auch Ergebnis der verhältnismäßig spärlichen Quellenüberlieferung. Denn für die Weimarer Landbevölkerung fehlen

13 M. Rainer Lepsius, Parteiensystem und Sozialstruktur. Zum Problem der Demokratisierung der deutschen Gesellschaft, in: Wilhelm Abel (Hrsg.), Wirtschaft, Geschichte und Wirtschaftsgeschichte. Festschrift zum 65. Geburtstag von Friedrich Lütge, Stuttgart 1966, S. 371-393.

14 Antonius Liedhegener, Christentum und Urbanisierung. Katholiken und Protestanten in Münster und Bochum 1830-1933, Paderborn 1997; ders., Katholisches Milieu in einer industriellen Umwelt am Beispiel Bochum. Strukturen und Entwicklungslinien 1830-1974, in: Matthias Frese/Michael Prinz (Hrsg.), Politische Zäsuren und gesellschaftlicher Wandel im 20. Jahrhundert. Regionale und vergleichende Perspektiven, Paderborn 1996, S. 545-596; Cornelia Rauh-Kühne, Anpassung und Widerstand? Kritische Bemerkungen zur Erforschung des katholischen Milieus, in: Detlef Schmiechen-Ackermann (Hrsg.), Anpassung, Verweigerung, Widerstand. Soziale Milieus, politische Kultur und der Widerstand gegen den Nationalsozialismus in Deutschland im regionalen Vergleich, Berlin 1997, S. 145-164; dies., Katholisches Milieu und Kleinstadtgesellschaft. Ettlingen 1918-1939, Sigmaringen 1991; dies., Katholisches Sozialmilieu, Region und Nationalsozialismus, in: Horst Möller/Andreas Wirsching/Walter Ziegler (Hrsg.), Nationalsozialismus in der Region. Beiträge zur regionalen und lokalen Forschung und zum internationalen Vergleich, München 1996, S. 213-236; Weichlein, Sozialmilieus; vgl. auch Doris Kaufmann, Katholisches Milieu in Münster 1928-1933. Politische Aktionsformen und geschlechtsspezifische Verhaltensräume, Düsseldorf 1984. 
uns oft jene aufschlußreichen Quellen, die wir der Überwachungsmanie des totalitären NS-Staates verdanken und die von dem Projekt „Bayern in der NS-Zeit“ mit viel Gewinn herangezogen wurden. Für die Weimarer Zeit am intensivsten hat sich daher wohl nicht zufällig die von dieser Art Quellenproblematik unbelastete historische Wahlforschung mit dem katholischen Land befaßt ${ }^{15}$.

Eine Ausnahme von der "Stadtlastigkeit“ der neueren, sozialgeschichtlich orientierten Katholizismusforschung bildet die eingangs zitierte vorzügliche mentalitätsgeschichtliche Studie zum konfessionellen Verhalten der Landbevölkerung Badens von Alfred Wahl. Auch, aber nicht nur wahlhistorische Fragen stellt die methodisch in manchen Aspekten vergleichbare Untersuchung Peter Exners über drei westfälische Dörfer im Modernisierungsprozeß des 20. Jahrhunderts, wenngleich sie stärker agrarsoziologisch interessiert ist und die lebensweltliche Ebene beleuchtet.

Steht in diesen Arbeiten das sozialgeschichtliche Interessen an historischen Transformationsprozessen von langer Dauer im Vordergrund, so konzentrieren sich die Arbeiten von Zdenek Zofka über den schwäbischen Landkreis Günzburg und von Oded Heilbronner zum südlichen Schwarzwald auf die relativ kurze Phase des Aufstiegs des Nationalsozialismus und der nationalsozialistischen Gleichschaltung ${ }^{16}$. Zu konstatieren ist mithin ein unverbundenes Nebeneinander von nur wenigen Arbeiten, von denen einige sich für Beharrung und Wandel konfessionell-ländlicher Lebensweisen und Einstellungsmuster interessieren, andere dagegen die politische Zäsur 1933 und wieder andere den Aufstieg der NSDAP oder aber Politik, Kultur und Organisationsgeschichte der KPD behandeln. Die speziellen Problemlagen ländlich-katholischer Gebiete kamen dabei selbst in jenen neueren Arbeiten zu kurz, die sich sozialgeschichtlicher Methoden bedienen, um die Verschränkung von Milieu und Politik zu untersuchen.

Noch immer fehlt es für die katholische Agrargesellschaft an methodisch komplexen Arbeiten, die dem Zusammenhang von Sozialstruktur, Agrarverfassung, landwirtschaftlicher Konjunktur, regionaler politischer Tradition, Milieu und Politik nachgehen und die so den Einbruch oder die Abwehr radikaler Parteien nachvollziehbar machen könnten. Politisches Verhalten müßte vor dem Hintergrund der kulturellen Praxis in dörflichen Lebensweisen verständlich gemacht werden ${ }^{17}$. Was aber wissen wir bislang über die Konfrontation der katholischländlichen Lebenswelt mit den radikalen Parteien der Weimarer Republik? Gehen

15 Einen Überblick über die historische Wahlforschung mit ausführlichem Literaturteil gibt: Falter, Hitlers Wähler; ebenfalls stark an der Entwicklung des Wahlverhaltens orientiert ist der Abriß, den Frank Nienhaus von seinem Dissertationsprojekt publiziert hat: ders., Transformations- und Erosionsprozesse des katholischen Milieus in einer ländlich-textilindustrialisierten Region: Das Westmünsterland 1914-1968, in: Frese/Prinz (Hrsg.), Politische Zäsuren, S. 597-629.

16 Wahl, Confession; Peter Exner, Ländliche Gesellschaft und Landwirtschaft in Westfalen 19191969, Paderborn 1997; Zdenek Zofka, Die Ausbreitung des Nationalsozialismus auf dem Lande. Eine regionale Fallstudie zur politischen Einstellung der Landbevölkerung in der Zeit des Aufstiegs und der Machtergreifung der NSDAP 1928-1936, München 1979.

17 Vgl. die methodische Skizze in der dem ländlich-protestantischen Milieu gewidmeten Studie von Pyta, Dorfgemeinschaft, S. 28. Die als "großflächige Synthese“ angelegte Untersuchung wird dem selbst erhobenen Anspruch auf Interdisziplinarität allerdings nicht in allen Teilen gerecht. Vermutlich können nur kleinräumig konzipierte Studien einen solchen integrativen Ansatz durchhalten, der m. E. auch Anleihen bei der Kommunikationswissenschaft zu machen hätte. 
wir zunächst der Frage nach, unter welchen Bedingungen KPD und NSDAP auf dem Lande die Stimmen katholischer Wähler und Wählerinnen erhielten und welche Hemmnisse sich dem Aufstieg dieser Parteien hier entgegenstellten ${ }^{18}$.

Nach den Ergebnissen Alfred Wahls zu Baden gelang der KPD früher als der NSDAP oder ihren völkischen Vorläufern der Einbruch in das katholische Land. Erstmals konnten die Kommunisten im Mai 1924 in nennenswertem Maße Wählerstimmen der Landbevölkerung gewinnen. Das war bei jener ersten Reichstagswahl dieses Jahres, die mitten in die Stabilisierungskrise fiel und den Inflationswirren der vorausgehenden Jahre unmittelbar folgte. Das Rekrutierungsgebiet der KPD beschränkte sich jedoch auf Wohngemeinden mit nennenswert industrieller Erwerbstätigkeit. Dabei war auffällig, daß die Partei genau die Ausbreitungswege nahm, die vor 1914 die SPD geebnet hatte. Gleichgültig, ob es sich um protestantisch oder katholisch dominierte Gegenden handelte, war die kommunistische Partei in Weimar zuerst dort erfolgreich, wo im Kaiserreich die SPD Hochburgen ausgebaut hatte. Dabei handelte es sich meist um Arbeiterdörfer im Umfeld ländlicher Textil- und Tabakindustriebetriebe, wie sie im Wiesental im Kreis Lörrach oder in der Rheinebene im Hinterland Offenburgs häufig vorkamen. In solchen Gemeinden, in denen sich die Arbeiterbewegung schon früh hatte etablieren können, war es häufig parallel zum Ausbau der Organisationsstrukturen eines katholischen Milieus gekommen; den katholischen Organisationen gelang es offenbar nicht, das einmal etablierte sozialistische Arbeitermilieu wieder zu verdrängen, das sich im Gegenteil - bedingt durch wirtschaftliche und gesellschaftliche Krisen - nach 1918 partiell radikalisierte.

Bei der Reichstagswahl 1928, als der konjunkturelle Aufstieg der Weimarer Wirtschaft seinen Höhepunkt erreicht hatte, die Textil- und Tabakindustrie jedoch bereits in einer schweren Krise steckten, konnte die KPD in diesen industrienahen Dörfern ihre Stellung weiter ausbauen. In 17 Landgemeinden Badens erzielte sie mehr als 25 Prozent der Stimmen, mehr als das doppelte des Reichsdurchschnitts also. Bei fast der Hälfte dieser KPD-Hochburgen in badischen Landgemeinden handelte es sich um katholische Gemeinden, die insgesamt jedoch im Freistaat Baden bei weitem in der Mehrzahl waren. Es ergab sich also hinsichtlich der Verteilung kommunistischer Hochburgen auf dem Lande eine deutliche Unterrepräsentation der katholischen Gemeinden.

Unter dem Durchschnitt lag auch der Anteil der KPD-Mitglieder in der Textilund Tabakindustrie, also in jenen Industriebranchen, die sich vereinzelt inmitten ländlich-katholischer Gegenden abseits industrieller Ballungszentren angesiedelt hatten. Die Möglichkeit landwirtschaftlichen Nebenerwerbs, ein in der Textil- wie Tabakindustrie hoher Anteil weiblicher Erwerbstätigkeit und, damit verbunden, ein geringer gewerkschaftlicher Organisationsgrad scheinen jene Faktoren gewe-

18 Vgl. für den agrarisch dominierten Amtsverband Velen-Ramsdorf im Westmünsterland: Norbert Fasse, Katholiken und NS-Herrschaft im Münsterland. Das Amt Velen-Ramsdorf 1918-1945, Bielefeld 1996. Es handelt sich, obwohl dieses Buch ein kommunales Auftragswerk ist, um eine methodisch anspruchsvolle, mit dichtem Quellenmaterial gearbeitete Lokalstudie zu einer Zentrumshochburg mit im März 1933 jedoch beachtlicher nationalsozialistischer Minderheit (20 Prozent im Amt Velen, 28,3 Prozent im sozialstrukturell inhomogeneren Amt Ramsdorf) und Höchstanteilen der KPD im November 1932 von 2,2 Prozent im Amt Velen und 5,2 Prozent im Amt Ramsdorf. Ebd., S. 42 u. 131. 
sen zu sein, die einer politischen Radikalisierung der Arbeiterschaft des katholischen Landes entgegenwirkten ${ }^{19}$.

Auch die NSDAP und ihre völkischen Vorläufer stießen bei ihrer Ausbreitung in katholischen Regionen auf strukturelle Hemmnisse. Bei der Mai-Wahl 1924 zeigte sich das besonders deutlich, denn in konfessionell gemischten Gebieten Badens erfaßte die nun erstmals zu beobachtende Radikalisierung durch die politische Rechte nur die evangelischen Dörfer. Selbst dort, wo katholische Gemeinden an völkisch-protestantische Hochburgen angrenzten, machte die Radikalisierung des politischen Verhaltens an der Dorfgrenze Halt. Wahl interpretiert diese $\mathrm{Zu}$ rückhaltung gegenüber einer in der politischen Parteienlandschaft neuen Gruppierung als Bestätigung für die jeder Neuerung abholde katholische Mentalität: "Comme à l'ordinaire, les catholiques firent preuve d'une meilleure résistance à toute nouveauté, alors que les protestants montrèrent leur instabilité en matière électorale et leur tendance à adopter rapidement les nouvelles tendances qui se constituaient" 20 . Auch 1928 noch trat die NSDAP bei den Reichstagswahlen in Baden als die Partei evangelischer Bauern auf.

Ihre Ausbreitung in dominant katholische Gemeinden startete die NSDAP in Baden erst in der Weltwirtschaftskrise. 1929 bei der Landtagswahl, bei der die Hitler-Partei ihr Vorjahresergebnis bei der Reichstagswahl mehr als verdoppeln konnte und landesweit 7 Prozent der Stimmen erlangte, waren es nochmals protestantische Landgemeinden, in denen sich die Nationalsozialisten hatten etablieren können. Schnell gelang es ihnen danach jedoch, von hier aus Brückenköpfe in das katholische Land zu errichten. Im September 1930 schnitt die NSDAP bei der Reichstagswahl in Baden noch besser ab als im Reich und erzielte 19,2 Prozent der Stimmen. Ihr Verbreitungsgebiet erstreckte sich nun auf alle ländlich-protestantischen Kreise des Landes. Sie verfügte jetzt im stärker protestantisch geprägten Norden ebenso wie im dominant katholischen Süden über Hochburgen und hatte sich von dort auch auf etliche katholische Gebiete ausgedehnt.

Schwerpunkte des nationalsozialistischen Erfolgs im katholisch-ländlichen Baden waren Gemeinden in konfessionell gemischten Landkreisen. Die mentalen Vorbehalte, die dort in der Frühphase des Aufstiegs des Nationalsozialismus die Ausbreitung der NSDAP über die protestantischen Ortsgrenzen hinaus behindert hatten, schienen beseitigt. In zahlreichen katholischen Gemeinden war nun der Damm gebrochen, ohne daß die bislang vorliegende Forschung es zuließe, $\mathrm{Zu}$ sammenhänge zwischen Sozialstruktur, Milieukultur und Wahlverhalten dieser Gemeinden herzustellen. Wenn es stimmt, was jüngst - gestützt auf eine umfangreiche Datenanalyse von 42000 frühen NSDAP-Mitgliedern - der amerikanische Soziologe William Brustein vertreten hat, so wären die z. T. erheblichen Diskrepanzen der NSDAP-Wahlerfolge in konfessionell und sozio-strukturell weitgehend übereinstimmenden Untersuchungseinheiten hauptsächlich auf unterschiedliche materielle Interessenlagen zurückzuführen ${ }^{21}$. Angesichts der regional bedeutenden Unterschiede des bäuerlichen Erbrechts, der damit zusammenhän-

19 Vgl. Thomas Kurz, Feindliche Brüder im deutschen Südwesten. Sozialdemokraten und Kommunisten in Baden und Württemberg von 1928 bis 1933, Berlin 1996, S. 40.

20 Wahl, Confession, S. 1164.

21 Brustein, Logic of Evil, S. 102. 
genden landwirtschaftlichen Besitzgröße, der Bodenqualität, Rentabilität, Produktionsweisen, Absatzchancen usw. scheint es plausibel, gerade für die Untersuchung des politischen Verhaltens der Landbevölkerung diese These zu überprüfen.

Auffällig ist in Baden die Konzentration katholischer NS-Hochburgen im Obst-, Wein- und Tabakbaugebiet Mittelbadens sowie im Schwarzwald, wo neben Viehhaltung und Milchwirtschaft traditionell die Holzwirtschaft von existentieller Bedeutung war. Das waren Gebiete, die unter dem seit 1924 fortschreitenden Preisverfall für Agrarprodukte und seit 1930 auch für Bauholz in extremer Weise zu leiden hatten, während sie vom Agrarprotektionsmus für Getreideprodukte der ostelbischen Landwirtschaft in keiner Weise profitierten. Wie drastisch sich z. B. für Weinbauern der Preisverfall auswirkte, verdeutlicht das Beispiel der Winzer im mittelbadischen Ort Oberschopfheim, die 1930 nur noch zwischen 71 und 57 Prozent des Verkaufspreises einnahmen, den sie 1927 erzielt hatten. Landwirte, die Tabak anbauten, erlitten einen ähnlichen Einnahmeverlust. Frostschäden und Unwetter führten darüber hinaus in derselben Region viermal hintereinander zu Mißernten22. Die Notlage der obst-, wein- und tabakanbauenden Landwirte verstärkte sich schließlich noch durch fiskalische Maßnahmen der Regierung Brüning, wie z.B. die Zigarettenpapier- und Getränkesteuer, so daß es häufig zu Zwangsversteigerungen landwirtschaftlicher Anwesen kam. Die regionale Presse kündigte zu Beginn des Jahres 1933 an, wenn die Agrarkrise weitere drei Jahre anhalten werde, würden noch viele Bauern der Gegend ihren Besitz verlieren ${ }^{23}$.

In der betreffenden Region waren 1930 NSDAP-Ergebnisse von örtlich mehr als 30 Prozent in katholisch dominierten Gemeinden keine Seltenheit ${ }^{24}$, während es jedoch auch hier nicht an Beispielen dafür fehlte, daß unmittelbar benachbarte Ortschaften vergleichbarer Größe, Sozial- und Konfessionsstruktur sich als fast vollkommen resistent erwiesen. Im Juli 1932, als die NSDAP mit 37 Prozent der Stimmen reichsweit stärkste Partei wurde, gelang es der katholischen Zentrumspartei nicht selten, sich gerade in braunen Hochburgen zu behaupten und einen weiteren Zuwachs der Hitler-Partei zu verhindern.

Aber bei der Reichstagswahl im Juli gab es allein in Baden 96 katholische Dörfer, in denen die NSDAP mehr als die Hälfte der Wähler und Wählerinnen hinter sich brachte. Ausnahmslos handelte es sich um sehr kleine Ortschaften mit weniger als 400 Einwohnern, um einen Gemeindetyp, der gleichzeitig das Gros der Zentrumsanhängerschaft stellte. Freilich zeigt ein Blick zurück auf die Wahlergebnisse 1912 und 1924 eine Gemeinsamkeit der politischen Tradition solch katholischer brauner Hochburgen: Die NSDAP erreichte auf dem katholischen

22 Für die katholische Gemeinde Oberschopfheim im politisch stark radikalisierten gemischt konfessionellen Landkreis Lahr: Walter Rinderle/Bernard Norling, The Nazi Impact on a German Village, Kentucky 1993, S. 73; Heilbronner, Achillesferse, S. 24-27.

23 Wiedergabe des Lahrer Anzeigers nach Norling/Rinderle, Nazi Impact, S. 76.

24 Beispiele: Oberschopfheim, Kreis Lahr: 28 Prozent; Oberhamersbach, Kreis Offenburg/Ortenau: 31,7 Prozent; Hinterzarten, Kreis Neustadt (Südschwarzwald): 31,8 Prozent. Vgl.: Rinderle/Norling, Nazi Impact; S. 102; Karl-August Lehmann, Die Zeit der Weimarer Republik und des Nationalsozialismus in Oberhamersbach, in: Die Ortenau 67 (1987), S. 399-420, hier S. 411; Heilbronner, Achillesferse, S. $76 \mathrm{f}$. 
Land dort ihre besten Ergebnisse, wo schon im Kaiserreich ein erheblicher Anteil der Wähler sich vom Zentrum abgewandt hatte, und wo sich dieser Trend, von dem zunächst Liberale, Konservative oder Badischer Landbund profitiert hatten, nach 1918 fortsetzte. Wahl folgert aus seinen Ergebnissen: „Il est clair que les électeurs nazis se recrutaient dans les familles où l'on avait plus voté pour le Zentrum dès le Reich wilhelmien". ${ }^{25}$

Über die Ursachen und Motive dieser katholischen Dissidenten können wir beim gegenwärtigen Forschungsstand lediglich spekulieren. Für die frühe Abwendung katholischer Wähler von der Zentrumspartei und ihre Hinwendung zu einer liberalen Partei oder zum Badischen Landbund kommen agrarpolitische Beweggründe ebenso in Frage wie weltanschauliche Dispositionen, religiöse Traditionen und antiklerikale Einstellungen, die wiederum auf lebensweltliche Erfahrungszusammenhänge zu befragen wären.

Festgehalten zu werden verdient, daß politische Traditionen dörflicher $\mathrm{Ge}$ meinschaften ihre Empfänglichkeit oder Resistenz gegenüber politischem Radikalismus ganz erheblich beeinflußten. Es gab offenbar so etwas wie eine langfristig wirkende kulturelle Imprägnierung, die - je nachdem - Resistenz oder Radikalisierung begünstigte. Agrarsoziologische Faktoren wie das Realteilungsrecht und das katholische Milieu destabilisierende Nachwirkungen kirchlich-liberaler Einflüsse wie des Wessenbergianismus ${ }^{26}$ scheinen Einfluß auf die Ausbildung solcher politischer Traditionen gehabt zu haben. Die Erforschung des politischen Verhaltens sollte sich daher auf einen entsprechend lang bemessenen Zeitraum erstrecken.

Und keinesfalls darf sie sich auf wahlhistorische Betrachtungen beschränken. Um den komplizierten Zusammenhang zwischen Sozialstruktur, ökonomischen Interessen, religiös-weltanschaulicher Prägung, ritualisierter Alltagskultur, Milieubindung, kirchen- und parteipolitischen Strategien und politischem Verhalten aufhellen zu können, bietet die ländliche Gesellschaft mit ihren vergleichsweise überschaubaren Strukturen und Kommunikationsbedingungen gute Voraussetzungen, wenn auch eine schwierige Quellenbasis. Doch bei gut überlegter Auswahl der Untersuchungseinheiten und mit methodischem Einfallsreichtum kann das Ineinandergreifen von kirchlichem Leben, Politik und Alltag, die subkulturelle Einbindung der Politik, hier mit besonderem Gewinn studiert werden.

Damit würde ein wichtiges Forschungsdesiderat erfüllt, denn wie wenig wir bislang über die Zusammenhänge der politischen und gesellschaftlichen Radikalisierung der Weimarer Zeit wissen, hat gerade die Kontroverse um die Ergebnisse einer neuen, stärker an sozialgeschichtlichen Fragen als an Parteistrategie und Organisationsgeschichte interessierten KPD-Forschung gezeigt ${ }^{27}$. Der von Klaus-

25 Wahl, Confession, S. 1233.

26 Der im Sinne der katholischen Aufklärung tätige Theologe Ignaz Heinrich Freiherr von Wessenberg war 1814 auf päpstliche Weisung hin von seinem Amt als Generalvikar des Bistums Konstanz entlassen worden. In der Folgezeit trat er besonders als publizistischer Kritiker des Ultramontanismus in Erscheinung.

27 Vgl. Andreas Wirsching, „Stalinisierung“ oder entideologisierte "Nischengesellschaft" ? Alte Einsichten und neue Thesen zum Charakter der KPD in der Weimarer Republik, in: Vierteljahrshefte für Zeitgeschichte 45 (1997), S. 449-466; mit bibliographischen Angaben zu weiteren Diskussionsbeiträgen die Replik von Klaus-Michael Mallmann, Gehorsame Parteisoldaten oder Eigensinnige 
Michael Mallmann behauptete Gegensatz von Milieu und Avantgarde hat für die ländliche Gesellschaft besondere Plausibilität, ist - wegen der schwachen Repräsentanz der KPD speziell auf dem katholischen Land - bislang aber noch kaum detailliert untersucht worden. Welche Einflüsse und Faktoren waren es, die dort zur Parteinahme für die Kommunisten stimulierten, sei es durch anonym vollzogene Stimmabgabe, sei es durch Parteibeitritt oder sogar durch Agitationsarbeit und symbolisch expressive Bekenntnisse? Bildeten innerdörfliche soziale Spannungen - etwa zwischen verschiedenen Besitzschichten oder zwischen Eingesessenen und Zugewanderten, Alten und Jungen - die soziale Voraussetzung für eine politische Differenzierung, die zur Etablierung von KPD-Hochburgen in katholischen Arbeiterdörfern führte?

Schließlich und vor allem: wie war es in den „KPD-Hochburgen“ - wenn man denn im Kontext dörflich-katholischer Lebenswelt von solchen reden kann - um die „Eindringtiefe“ kommunistischer Ideologie, um die Existenz gar einer kommunistischen Identität bestellt? Wie radikal war das Rekrutierungspotential der KPD auf dem Lande, wenn wir etwa erfahren, daß in ganz Baden im Jahr 1927 nur 21 Prozent der Mitglieder aus der Kirche ausgetreten waren 28 ? Kirchlichkeit und KPD-Mitgliedschaft scheinen - zumal auf dem katholischen Lande - keine von vornherein unvereinbaren Verhaltensweisen gewesen zu sein. Viele Kommunisten, zumindest aber die Mehrzahl der KPD-Wähler und vor allem Wählerinnen, dürften dort die kirchlichen Riten von Taufe und Begräbnis absolviert haben. Ja, es gibt selbst Belege dafür, daß Genossen durch die Partei gemaßregelt werden mußten, weil sie sich bei Prozessionen als Vorbeter betätigt hatten ${ }^{29}$.

Wir kennen zahlreiche Äußerungen kommunistischer Parteifunktionäre, die belegen, wie schlecht die politische Arbeit der KPD unter Bauern beider christlicher Konfessionen fruchtete. Und das galt auch für Regionen, in denen die Lage der Landwirtschaft katastrophal und Zwangsversteigerungen von Höfen an der Tagesordnung war. ${ }^{30}$ In einem von der badischen politischen Polizei verfaßten Bericht vom September 1931 wird von enormer Aktivität der KPD berichtet, die nach den polizeilichen Beobachtungen allerdings weit hinter derjenigen der NSDAP zurückblieb. Die kommunistische Gefahr wurde als nicht bedeutend eingeschätzt, und ausdrücklich hieß es: „Der kommunistische Einfluß unter der Bauernschaft dürfte [...] trotz aller Bemühungen noch nicht sehr tiefgreifend sein" ${ }^{31}$. Überall auf dem Lande bestand ein „tiefsitzendes bäuerliches Mißtrauen“ gegenüber der kommunistischen Haltung zum Privatbesitz an Grund und Boden, das „nicht durch die Verkündigung noch so bauernfreundlich aufgemachter Agrarprogramme auszuräumen [war], zumal die Zwangskollektivierung im kommuni-

Akteure? Dic Weimarer Kommunisten in der Kontroverse - eine Erwiderung, in: Vierteljahrshefte für Zeitgeschichte 47 (1999), S. 401-415.

28 Kurz, Feindliche Brüder, S. 40; zur Kirchenmitgliedschaft von Kommunisten in ländlich-evangelischen KPD-Hochburgen: Pyta, Dorfgemeinschaft, S. 281.

29 Ulrich Neuhäußer-Wespy, Die KPD in Nordbayern 1919-1933, Nürnberg 1981, S. 117.

so Vgl. die Erinnerungen von Max Faulhaber, Mitglied des Agrarausschusses der badischen KPD und bis 1933 als Agitator tätig: ders., "Aufgegeben haben wir nie...". Erinnerungen aus einem Leben in der Arbeiterbewegung, herausgegeben von Peter Fäßler u. a., Marburg 1988, S. $106 \mathrm{ff}$.

31 Kurz, Feindliche Brüder, S. 356. 
stischen Musterland Sowjetunion die Zweifel an der Aufrichtigkeit kommunistischer Bauernpropaganda noch nährte" ${ }^{32}$.

Aber auch bei ländlichen Arbeitern scheint der Kommunismus in vielen Fällen ein Oberflächenphänomen gewesen zu sein, Ausdruck des Protests gegen die Verschärfung ihrer wirtschaftlich ohnehin prekären Situation hier, Demonstration des weltanschaulichen Dissenses gegen eine von kirchlichen Normen geprägte Dorfhierarchie da. Häufiger jedoch scheint es bei den örtlichen KPD-Anhängern „breite Übereinstimmung mit den Moralvorstellungen des lokalen Umfeldes“ gegeben zu haben. Ihren Lebensstil richteten sie stärker als am kommunistischen Programm an lokalgesellschaftlichen Maßstäben von Respektabilität aus, deren Inbegriff auch in Arbeitergemeinden das eigene Haus war. Klaus-Michael Mallmann hat daher zu Recht die „relative Eigengesetzlichkeit der lokalen Gesellschaft" betont ${ }^{33}$.

Sie gilt es auch zu berücksichtigen, wenn man den Ursachen des Erfolgs der NSDAP auf dem katholischen Land nachgehen will. Wie erklärt sich die unterschiedliche Anfälligkeit einzelner Dorfgemeinschaften für die radikale Herausforderung von rechts? Welche programmatischen Versprechungen, propagandistischen Ereignisse oder kommunikativen Strukturen beeinflußten die Wahl- oder gar Beitrittsentscheidung, und welchen Stellenwert hatten ideologische Komponenten wie der von der NSDAP verfochtene radikale Antisemitismus oder kirchenfeindliche Bestrebungen? Nach Oded Heilbronners Beobachtungen gingen den Wahlerfolgen der NSDAP in katholischen Schwarzwaldgebieten 1930 kaum bemerkbare propagandistische Anstrengungen voraus. Die Partei war dort angeblich weder publizistisch noch agitatorisch präsent und erzielte dennoch weit überdurchschnittliche Wahlergebnisse. Ein Erfolg, den Heilbronner ohne konkreten Nachweis, aber mit einiger Plausibilität dem Einfluß lokaler Meinungsführer zuschreibt, die in Gemeinden ohne organisatorische Verfestigung eines katholischen Milieus gewissermaßen vom Stammtisch aus zu wirken vermochten. Offen bleibt allerdings, wie, über welche Informationskanäle die Opinion makers mit dem Programm der NSDAP in Berührung kamen ${ }^{34}$. Entscheidend zugunsten der NSDAP könnte sich die in Baden schon früh geglückte Unterwanderung des landwirtschaftlichen Verbandswesens ausgewirkt haben. Wolfram Pyta hat darauf hingewiesen, wie wirkungsvoll der von Walter Darré gesteuerte Agrarpolitische Apparat der NSDAP hier das Geschäft der Nationalsozialisten besorgte. Am frühesten und erfolgreichsten gelang es dieser Organisation in Baden, einen bedeutenden agrarischen Interessenverband zu infiltrieren. Durch die Zusammenarbeit der NSDAP mit der regionalen Organisation des Reichslandbunds, dem Badischen Landbund, gab es bereits 1931 in allen 40 Amtsbezirken des Landes von der NSDAP benannte landwirtschaftliche Bezirksfachberater, die sich ihrerseits be-

32 Pyta, Dorfgemeinschaft, S. 280. Dieser Befund kann gewiß auf das katholische Land übertragen werden, wo die katholische Geistlichkeit dazu beitrug, die christentumfeindliche Politik in der Sowjetunion publik zu machen und bei dieser Gelegenheit auch die Zwangskollektivierung kritisierte. Horst W. Hetzer, Deutscher Katholizismus und „Bolschewismusgefahr" bis 1933, in: Historisches Jahrbuch der Görres-Gesellschaft 113 (1993), S. 355-387.

33 Mallmann, Kommunisten, S. 294.

34 Heilbronner, Achillesferse, S. 14; auch Pyta sieht in der Autorität dörflicher Meinungsführer ein Erklärungsmuster für den politischen Prozeß auf dem Lande. Ders., Dorfgemeinschaft, S. 83 ff. 
mühten, vor Ort „politisch zuverlässige“ Landwirtschaftliche Ortsgruppenfachberater und Vertrauensleute $\mathrm{zu}$ installieren ${ }^{35}$. Wie erfolgreich sie dabei waren, dürfte in erster Linie davon abhängig gewesen sein, wie stark der Badische Landbund vor Ort war. Überall, wo noch ein intaktes katholisches Milieu bestand, waren die Landwirte im Badischen Bauernverein organisiert, einer katholisch-berufsständischen Organisation, die durch ihre Nähe zur Zentrumspartei, zur katholischen Kirche und deren weltanschauliche Gegnerschaft zum Nationalsozialismus einen wirksamen Schutz gegen radikale Tendenzen der ländlichen Wählerschaft darstellte ${ }^{36}$. In evangelischen Gebieten aber und dort, wo sich in manchen katholischen Gegenden Südbadens agrarische Berufsgruppen schon im Kaiserreich vom Zentrum und seinen berufsständischen Organisationen abgewendet und konservativ oder liberal gewählt hatten, lagen traditionelle Domänen des Landbunds. Vieles deutet darauf hin, daß dieser Agrarverband entscheidenden Anteil daran hatte, die Landbevölkerung außerhalb des katholischen Milieus für die NSDAP zu gewinnen. Möglicherweise waren die lokalen Vertreter des Landbunds auch jene „Brückenköpfe“, von denen aus es schließlich 1932/33 gelang, wichtige Stützpunkte in genuin „katholischem Gebiet“ zu errichten.

Zwangsläufig schließt sich die Frage an, welche Interessen es hier waren, die der Radikalisierung von rechts in einer von ländlicher Lebensweise und kirchlichem Einfluß geprägten Region Vorschub leisteten? Waren es wirklich primär materielle, agrarpolitische oder wirtschaftliche Interessen, die den Nationalsozialismus in parochial strukturierten ländlichen Gebieten für seine Anhänger attraktiv machten ${ }^{37}$ ? Die Annahme, die weithin unverkennbar wirksame Resistenz des katholischen Landes gegenüber der NSDAP sei dem dort vorherrschenden Realteilungsrecht zuzuschreiben, wird man als monokausale Erklärung kaum akzeptieren. Aber festgehalten zu werden verdient: Wir wissen bisher wenig darüber, inwieweit es die NSDAP verstand, ihre Propaganda auf dem Lande auf die örtlichen Probleme, wirtschaftlich vorherrschenden Interessen und lebensweltlichen Bedürfnisse einzustellen. Daß sie sich in ihrer praktischen Politik als weitaus flexibler und viel weniger dogmatisch erwies als die zentral gesteuerte KPD ${ }^{38}$, darf jedenfalls als gewiß gelten.

So sind uns die Motivationslagen und Strategien derjenigen, die sich auf dem Lande von der herrschenden katholischen Partei ab- und der NSDAP oder KPD zuwandten, bislang weitgehend unbekannt geblieben. Sie zu erforschen, wäre nicht unwichtig, um vorschnelle Generalisierungen kritisch zu prüfen und die Faktoren politischer Radikalisierung kennenzulernen ${ }^{39}$.

35 Pyta, Dorfgemeinschaft, S. 362.

36 Vgl. Pyta, Dorfgemeinschaft, S. 217. Demnach verweigerten sich die katholischen Bauern Südund Westdeutschlands den 1931 vom Reichslandbund organisierten Steuerverweigerungsaktionen, obwohl sie kaum weniger heftig von der Agrarkrise heimgesucht waren als ihre protestantischen Berufskollegen im Norden und Osten des Reichs.

37 Vgl. Brustein, The Logic of Evil, S. 102; ähnlich Pyta, Dorfgemeinschaft, S. 201; Rinderle/Norling, Nazi Impact, S. 95.

38 Beispiele bei Kurz, Feindliche Brüder, S. 282f., 345, 348, 359, 379.

39 Als vorschnelle Generalisierung erscheint es mir, wenn unter Rückgriff auf die von Gabriel Almond und Sidney Verba vorgenommene Unterscheidung verschiedener politischer Kulturen aus dem Idealtypus einer durch ausgeprägte Politikferne geprägten Kultur des Dorfes kurzerhand ein Realtypus konstruiert wird. Das Politische drang oft in lokalistischer Aufmachung in lebenswelt- 
Dagegen lassen sich die Ursachen relativer Stabilität benennen. Die außerordentliche, von keiner anderen Partei des Weimarer Parteiensystems erreichte Beständigkeit des Zentrums und seiner Schwesterpartei, der Bayerischen Volkspartei, war Ausdruck der weltanschaulichen Geschlossenheit und sozialen Kohäsion des katholischen Milieus. In kleinen Gemeinden mit unter 1000 Einwohnern, in denen industrielle Erwerbsarbeit keine nennenswerte Rolle spielte, war sie am ausgeprägtesten.

Hier, wo es an sozialer Dynamik und innerdörflicher sozialer Ausdifferenzierung fehlte, bedurfte es - anders als in den Städten - zur Wahrung der Zentrumstreue noch nicht einmal der organisatorischen Abstützung des Milieus durch ein katholisches Vereinsnetz. Dort indessen, wo sich - meist schon um die Jahrhundertwende - Industriebetriebe angesiedelt hatten und der Zuzug auswärtiger Arbeitskräfte zur Entstehung sozialdemokratischer Organisationskerne geführt hatte, war es meist auf katholischer Seite zur Entstehung einer dichten, alle Lebensbereiche abdeckenden Vereinskultur und zur aggressiven Abschottung gegen die Sozialdemokratie gekommen. Die Reichweite der katholischen Parteien war in solchen Arbeiterdörfern geringer als in der Mehrzahl der Bauerndörfer, aber die auf niedrigerem Niveau erlangte Stabilität von Zentrum bzw. BVP wurde in den Jahren nach 1918 durch Radikalisierungstendenzen der Weimarer Gesellschaft doch kaum beeinträchtigt. Denn in größeren wie in kleinen Gemeinden, im ländlichen wie im städtischen Kontext, war das katholische Milieu charakterisiert durch eine brauchgestützte, das Alltagsleben ritualisierende Kultur. Individuelle Lebensführung und katholisches Wert- und Normensystem wurden ständig miteinander vermittelt, das Individuum so schon auf einer vorbewußten Ebene in das katholische Normen- und Glaubenssystem integriert. Das politische Verhalten war Bestandteil dieses Normensystems und soziale Kontrolle und Konformitätsdruck, die im Dorf besonders wirksam waren, begünstigten seine Einhaltung.

Ökonomische Rückständigkeit und daraus resultierende Abschottungstendenzen des katholischen Milieus hatten somit die paradoxe Wirkung, daß die subkulturelle Einbindung der Politik, die auf der Makroebene die innere Nationsbildung und eine frühzeitige Parlamentarisierung Deutschlands verhindert hatte, an der Basis doch zugleich eine Schwelle gegen die Zerstörung der parlamentarischen Demokratie durch radikale, systemfeindliche Parteien bildete ${ }^{40}$. KPD wie NSDAP stießen - zumal auf dem Lande - in diesem Milieu bis Januar 1933 an schwer überwindbare Grenzen.

liche Zusammenhänge vor. Es bedarf daher subtiler Untersuchungsstrategien, um es als solches wieder erkennbar zu machen. Gabriel Almond/ Sidney Verba, The Civic Culture. Political Attitudes and Democracy in Five Nations, Princeton 1963.

40 Thomas Nipperdey, Religion im Umbruch. Deutschland 1870-1918, München 1988, S. 31. 